\title{
RELACIÓN ENTRE CONDUCTA Y ACTIVACIÓN DE ÁREAS CEREBRALES. EMPLEO de LA TÉCNICA DE AgNOR en Psicología Comparada*
}

\author{
RELATIONSHIP BETWEen BEHAVIOR AND ACTIVATION OF BRAIN AREAS. USING THE AgNOR \\ TECHNIQUE IN COMPARATIVE PSYCHOLOGY
}

\author{
Martín Miguel Puddington ${ }^{* *}$ y Rubén Néstor Muzio***
}

\begin{abstract}
*Trabajo financiado con el subsidio UBACYT-P0293BA de la Universidad de Buenos Aires (UBA), el PICT 2243 de la Agencia Nacional de Promoción Científica y Tecnológica (ANPCYT) y el PIP 0893 del Consejo Nacional de Investigaciones Científicas y Técnicas (CONICET), otorgados al Dr. Rubén N. Muzio.

**Doctor en Psicología. Becario Postdoctoral del Consejo Nacional de Investigaciones Científicas y Técnicas (CONICET), Miembro del Grupo de Aprendizaje y Cognición Comparada del Laboratorio de Biología del Comportamiento (IBYME) y Profesor Investigador de la Facultad de Psicología de la Universidad de Buenos Aires (UBA).

E-Mail: mmpudington@gmail.com

***Doctor en Ciencias Biológicas. Miembro de la Carrera del Investigador Científico del Consejo Nacional de Investigaciones Científicas y Técnicas (CONICET) y Profesor Asociado Regular de la Facultad de Psicología de la Universidad de Buenos Aires (UBA). Director del Grupo de Aprendizaje y Cognición Comparada del Laboratorio de Biología del Comportamiento (IBYME).E-Mail: rnmuzio@gmail.com Laboratorio de Biología del Comportamiento. Grupo de Aprendizaje y Cognición Comparada. Instituto de Biología y Medicina Experimental (IBYME). Vuelta de Obligado 2490 (C1428ADN). Ciudad Autónoma de Buenos Aires.

República Argentina.
\end{abstract}

\section{REsUmen}

Se presenta una revisión de distintos estudios que emplearon la técnica de AgNOR en el campo de las neurociencias del comportamiento. AgNOR es una técnica histológica que permite estudiar la activación cerebral de una región específica. Esta técnica ha sido utilizada en distintos grupos zoológicos que comprenden desde peces y anfibios hasta mamíferos, incluyendo actividad neural tanto en procesos normales como patológicos. En conjunto, la técnica de AgNOR tiene tres notables ventajas con respecto a otras técnicas histológicas como las inmunohistoquímicas. En primer lugar, su bajo costo. En segundo lugar, su robustez. Finalmente, la posibilidad de ser aplicada con facilidad a cualquier especie animal, siendo esto último particularmente relevante en el campo de la Psicología Comparada. Paradójicamente a pesar de estas ventajas, es una técnica poco conocida y empleada. El propósito de este artículo es contribuir a su difusión dentro de la comunidad de científicos del comportamiento.

Palabras clave: Conducta; Cerebro; Activación neuronal; Técnica de AgNOR; Psicología Comparada.

\section{ABSTRACT}

In this paper we review the use of $A g N O R$ technique in the field of behavioral neuroscience. AgNOR is a histological technique used to study the activation of a specific brain region. NORs (nucleolar organizer regions) are the cell nucleus compartments where part of the ribosomal synthesis occurs. When a neuron enhances its 
protein synthesis demand, for example during a learning process, NORs increase their size. Staining with silver nitrate, colors the cell nucleus in brown and the cell nucleolus in dark brown, nearly black. Thus, the size of the nucleus and the nucleolus can be easily measured in histological preparations treated with silver nitrate through any appropriated image processing software. In this manner, AgNOR technique consist in staining the tissue of subjects that have received different treatments and comparing the average size of the nucleolus of a particular brain region, which is an indirect marker of cellular activity. The use of AgNOR technique in the study of neural basis of learning, typically involves: (1) training animals in a particular task, (2) after acquisition, animals in the trained group along with animals from a control condition are sacrificed in order to obtain a brain sample, (3) brains of animals from both conditions are processed and compared. This procedure was successfully used to study telencephalic activation during spatial learning in fish (Carassius auratus). The task consisted in using visual cues to identify the correct exit from a trap. In the case of fish, the histological analysis showed an increase of the nucleolus size in the lateral pallium, proposed as homologous to the mammalian hippocampus, observing that this area is involved in spatial memory. In our laboratory this technique was also used to analyze the neural activity after spatial learning in the terrestrial toad Rhinella arenarum. We conducted several studies where dehydrated animals had to use visual cues to locate the correct position of a water source. In accordance with fish's data, our results indicated a higher activity of the hippocampal homologous region (the amphibian medial pallium), showing a high degree of functional equivalence. On the other hand, we also studied in toads the telencephalic activity during an aversive learning task. Here, it was observed an increase in the average of NORs size in the ventrolateral telencephalic region, the striatum (proposed as homologous to the mammalian amygdala), also showing a highly preserved functional equivalence. In mammals, AgNOR technique was used with different purposes, such as the study of changes in hippocampal activity during development in rats, the comparison of the basal activity of two regions of the rats hippocampus, the $\mathrm{CA} 1$ and the $\mathrm{CA} 3$, both strongly related with learning, or the the hippocampal activity in two different situations in which the liver activity was altered (by an artificial cholestasis, or by alcohol consumption). Finally, AgNOR technique was also used in the study of human brain activity of subjects that had committed suicide.

On the whole, the AgNOR technique has three distinct advantages over other histological techniques such as the immunohistochemical. First, their low cost. Second, their robustness. Finally, the fact that it can be easily applied to any animal species, which makes it particularly attractive to the field of the Comparative Psychology. But paradoxically, despite these advantages it is a poorly known and used technique. The aim of this paper is to contribute to the diffusion of the AgNOR technique in the community of behavioral scientists, showing that its use has provided a valuable contribution to research in different fields and animal species.

Key words: Behavior; Brain; Neuronal activation; AgNOR technique; Comparative Psychology.

\section{INTRODUCCIÓN}

\section{EL ROL DE LAS NEUROCIENCIAS EN LA PSICOLOGÍA}

Podría decirse que la Psicología es aque1la rama de la ciencia que busca explicar y predecir el comportamiento. Para ello los psicólogos observan la conducta en relación con las variables ambientales y hacen inferencias sobre los estados internos del organismo (McWilliams, 2015). De esta forma, una de las claves para poder predecir el comportamiento se relaciona con entender cómo es el procesamiento interno de la información disponible en un ambiente. Esto ha sido históricamente un gran problema para los psicólogos, ya que los estados internos no son directamente observables. En los albores de la Psicología existían dos posturas fuertes y contrarias frente a esta problemática. Una de ellas proponía la introspección como modo de 
estudio de los estados internos (Titchener, 1909). La otra simplemente negaba el problema limitando a la observación del comportamiento, sin teorizar sobre los procesos internos (Watson, 1913).

Hoy en día no hay dudas de que el cerebro es el lugar donde ocurre ese procesamiento de la información (Edelman \& Tononi, 2000) y por lo tanto comprender el funcionamiento del cerebro probablemente sea una condición necesaria para lograr teorías psicológicas precisas.

Las neurociencias son en sí mismas una verdadera interdisciplina. En neurociencias no es posible avanzar si no se es capaz de hacer una síntesis del análisis del comportamiento y además, es un campo que naturalmente convoca a especialistas de distintas disciplinas relacionadas, como psicólogos, médicos, biólogos, lingüistas, matemáticos, físicos, químicos, ingenieros y expertos en computación, entre otros (Bear, Connors \& Paradiso, 2006).

Una de las principales preguntas de las neurociencias, que ha estado presente desde un inicio es sobre la función específica de las distintas regiones cerebrales. El cerebro no funciona en forma homogénea sino que tiene áreas especializadas en funciones particulares (Penfield \& Rasmussen, 1950; Purves et al., 2004). Estudiar cuál es la función de cada región cerebral y cómo esas regiones están interconectadas, nos permite teorizar acerca de la forma en que se procesa la información del ambiente.

La forma más elemental y tradicional que se utiliza para conocer la función de una estructura cerebral es la observación de las consecuencias sobre la conducta de un individuo que ha sufrido una lesión. Por eso los estudios pioneros de las neurociencias se centraban en el estudio de pacientes con daño cerebral (ver por ejemplo el famoso caso de Phineas Gage - Harlow, 1868). Pero con el tiempo se han desarrollado estrategias más sofisticadas que, en paralelo con el avance tecnológico, permiten observar qué ocurre dentro del cerebro cuando se produce un determinado comportamiento. En este sentido, las técnicas de neuroimagen han ayudado a entender en detalle la morfología de muchos circuitos neuronales y las funciones asociadas. Aunque muchas de estas técnicas presentan gran resolución espacial (como la resonancia magnética), las mismas tienen como limitante su resolución temporal, ya que permiten un registro de actividad con una demora de algunos segundos y no en tiempo real (Bandettini, 2009). En forma complementaria, se ha intentado aplicar la teoría de la información y los métodos de procesamiento de señales para extraer en forma sistemática la compleja información contenida en los registros de actividad cerebral (Quian Quiroga \& Panzeri, 2009). Por otro lado, se desarrollaron métodos para evaluar los cambios conductuales y estructurales producidos por la exposición a agentes químicos, tanto en humanos como en otras especies animales (Tilson, 2000). Así, en el caso del estudio de conductas en modelos animales no humanos (aunque también en ciertos análisis de muestras en humanos), el abordaje con técnicas neurohistológicas constituye una gran ayuda a la hora de comprender los mecanismos que subyacen a los comportamientos (Rodríguez, López, Vargas \& Salas, 1998). Indirectamente estas técnicas brindan una evidencia de activación neuronal de las áreas que previamente han participado en la ejecución repetida de una conducta. De esta manera, tienen la ventaja de ser un indicador de dónde han ocurrido cambios plásticos en el cerebro asociados a ese comportamiento (García-Moreno, Cimadevilla, González-Pardo \& Arias, 2000).

En este artículo se presenta la técnica histológica de AgNOR, que permite visualizar las áreas que tuvieron activación cerebral en un período previo y medir la intensidad con que estuvieron en funcionamiento. Esta técnica, a pesar de sus notables ventajas, tiene una limitada difusión entre los estudiosos del comportamiento.

\section{APRENDIZAJE Y SÍNTESIS DE PROTEÍNAS}

Los fenómenos de aprendizaje que se observan a nivel comportamental como un cambio en la conducta, tienen un correlato fisio- 
lógico. Distintas estructuras cerebrales se encargan de procesar la información del ambiente y promover luego cambios en el comportamiento. Por ejemplo, en el caso del aprendizaje apetitivo se ha observado que las neuronas dopaminérgicas del área tegmental ventral (ATV - en inglés ventral tegmental area, VTA), se activan cuando reciben un refuerzo novedoso. Si ese refuerzo está asociado a un estímulo ambiental que lo predice, con los sucesivos ensayos, estas neuronas se activarán ante la aparición del estímulo predictor, antes de que aparezca el refuerzo (Schultz, Dayan \& Montague, 1997). Otro ejemplo clásico de plasticidad neuronal es el del núcleo basolateral de la amígdala (se suele usar la abreviación BLA). Existen neuronas en el BLA que responden a la estimulación aversiva. Para que se produzca un condicionamiento de la respuesta de miedo, estas neuronas refuerzan su conexión con otras que responden al estímulo condicionado (Campese et al., 2014, 2015; Gore et al., 2015; Le Doux, 2003; Ramirez et al., 2015).

Es evidente que para que se den estos cambios en el patrón de respuesta de ciertas neuronas, deberán ocurrir algunos cambios estructurales y fisiológicos dentro de las células. Existe una enorme cantidad de situaciones en las que se ha verificado que estos cambios se deben a la producción de nuevas proteínas. Por ejemplo, en una preparación in vitro de tejido cerebral proveniente del hipocampo de una rata, es posible inducir mediante electrodos una asociación entre dos neuronas. Este fenómeno es conocido como potenciación de largo plazo (PLP - en inglés long-term potentiation, LTP). Sin embargo, si se aplica un fármaco inhibidor de síntesis de proteínas (eg., anisomisina), tal asociación no se produce (Frey, Krug, Reymann \& Matthies, 1998). Cuando esta droga se aplica in vivo en el hipocampo de un animal previo al entrenamiento en una tarea espacial, se puede observar un deterioro en la adquisición de este aprendizaje (Naghdi, Majlessi \& Bozorgmehr, 2003). Si, en cambio, se aplica en el núcleo accumbens, uno de los blancos principales de las neuronas dopaminérgicas del área tegmental ventral (ATV), se observa un deterioro en el aprendizaje instrumental apetitivo (Hernandez, Sadeghian \& Kelley, 2002).

La síntesis de proteínas parece ser necesaria no sólo para la adquisición de un aprendizaje, sino también para otros aspectos del aprendizaje y la memoria. Por ejemplo, el uso de anisomisina administrada localmente en la amígdala puede afectar la reconsolidación de un aprendizaje aversivo (Nader, Schafe \& LeDoux, 2000). También se ha observado que la extinción se ve afectada por la infusión de esta droga en el hipocampo (Power, Berlau, McGaugh \& Steward, 2006).

Haciendo un análisis comparado de esta cuestión, se puede observar que la síntesis de proteínas en fenómenos de aprendizaje no es un evento que ocurre únicamente en mamíferos. Por ejemplo, también se ha utilizado la anisomisina en aves, observándose efectos disruptivos similares a los registrados en mamíferos (Freeman, Rose \& Sholey, 1995). Y lo mismo ocurre en la Aplysia sp., una especie de molusco marino filogenéticamente muy distante de los mamíferos (Castellucci, Blumenfeld, Goelet \& Kandel, 1989).

Si el aprendizaje requiere síntesis de proteínas en lugares específicos del cerebro, entonces se pueden identificar esas regiones clave, estudiando en qué áreas cerebrales se incrementa la síntesis de proteínas durante una tarea en particular.

\section{NEURONAS, NÚCLEOS Y NUCLEOLOS}

Las neuronas comparten las características generales del resto de las células del cuerpo. Poseen una membrana de fosfolípidos y proteínas, un citoplasma y un núcleo. El núcleo de las neuronas, al igual que de cualquier célula eucariota posee la información genética contenida en las moléculas de ADN. Esta información consiste entre otras cosas, en instrucciones para la construcción de proteínas. Para poder sintetizar una proteína, cualquier célula debe hacerlo en dos pasos, el primero se denomina transcripción y el segundo traducción. El primer paso, la transcripción, implica hacer una copia del fragmento de ADN 
de interés. Para ello interviene una serie de enzimas cuyo producto final es una molécula llamada $A R N$ mensajero. Es importante señalar que las proteínas son macromoléculas formadas por una cadena de compuestos químicos denominados aminoácidos $\mathrm{y}$, dado que existen en total 21 tipos de aminoácidos diferentes, se pueden obtener combinaciones de secuencias muy variadas. El ARN mensajero es el que contiene la información sobre el orden en que deben ser colocados esos aminoácidos, para formar una proteína en particular. El segundo paso, la traducción, implica decodificar la información contenida en el ARN mensajero para ubicar los aminoácidos en el orden correcto. Esta tarea es llevada a cabo por estructuras llamadas ribosomas (Alberts, Johnson, Lewis, Raff, Roberts \& Walter, 2008).

Dentro del núcleo existe una estructura que se denomina nucleolo, cuya función es transcribir ARN ribosomal para la posterior formación de los ribosomas. El nucleolo está organizado en torno a segmentos de ADN específicos llamados regiones organizadoras nucleares (en inglés, NORs, nucleolar organizer regions). Cuando una célula necesita sintetizar una gran cantidad de proteínas, deberá incrementar el número de ribosomas para poder hacer frente a esa demanda. En tales situaciones el tamaño del nucleolo dentro del núcleo comienza a incrementarse y en ocasiones, puede dividirse en dos. De esta manera, el tamaño del nucleolo es un indicador indirecto del nivel de síntesis de proteínas que está ocurriendo en esa célula (Raška, Koberna, Malínský, Fidlerová \& Mašata, 2004) y por lo tanto, de su grado de activación.

\section{TÉCNICA DE AgNOR}

\section{VISUALIZACIÓN DE LA ACTIVIDAD CELULAR MEDIANTE LA TÉCNICA DE AGNOR}

Existe un procedimiento que permite observar el tamaño de núcleos y nucleolos celulares que se denomina técnica de AgNOR (Ploton, Menager, Jeannesson, Himber,
Pigeon \& Adnet, 1986). Se trata de una técnica histológica (lo que implica un análisis postmortem del tejido del individuo) que utiliza para la tinción nitrato de plata. Como en los NORs existen proteínas con afinidad por la plata (o proteínas argirofílicas), este procedimiento permite visualizar específicamente estas estructuras. Dado que $\mathrm{Ag}$ es el símbolo químico de la plata, se la conoce entonces como técnica de AgNOR.

Esta técnica puede aplicarse a cualquier tipo de tejido, pero aquí toma relevancia su uso en el tejido nervioso a fin de analizar la actividad neuronal de una determinada región del cerebro. El tejido cerebral debe ser cortado en secciones de alrededor de 15 micrones de espesor, las cuales se montan en un portaobjetos para teñirlas con el nitrato de plata. Una vez que estas secciones están teñidas, el tejido se visualiza ópticamente con una coloración general amarronada. Al amplificar el preparado con un microscopio se podrá observar que los núcleos de las células llevan un tono marrón intenso, mientras que los nucleolos un marrón aún más oscuro (con un matiz casi negro). La Figura 1 muestra algunos ejemplos de fotografías (en este caso, en blanco y negro) de tejido cerebral de sapos (Rhinella arenarum) y ratas (Rattus norvegicus) teñidos mediante la técnica de AgNOR.

CUANTIFICACIÓN DE LA ACTIVIDAD CEREBRAL MEDIANTE LA TÉCNICA DE AGNOR

Para poder cuantificar la actividad de una región cerebral particular se selecciona una cantidad fija de núcleos, usualmente 80 , y se registran las siguientes dos variables: Por un lado, se contabiliza dentro de esos 80 núcleos cuántos presentan más de un nucleolo (ver ejemplo en la Figura 2) y se estima el número promedio de nucleolos por núcleo. Por otro lado, se mide con alguna herramienta informática de procesamiento de imágenes, la superficie del nucleolo y la superficie total del núcleo. Se calcula entonces para cada neurona, el porcentaje de área del núcleo ocupada por el nucleolo. Tomando el promedio de esta relación para todos los núcleos seleccio- 
nados, se obtiene el porcentaje relativo de área AgNOR para esa región particular.

Como se mencionó en el apartado anterior, si el área cerebral seleccionada en un individuo hubiera tenido previamente un incremento en la actividad de síntesis de proteínas asociada a un aprendizaje, se esperaría que la superficie del nucleolo se viera aumentada respecto a la actividad de esa misma área en un sujeto control.

\section{EMPLEO DE LA TÉCNICA DE AGNOR}

\section{ANTECEDENTES EN ESTUDIOS CON MODELOS ANIMA- LES NO HUMANOS}

El uso más difundido de esta técnica en Psicología Experimental es su aplicación a modelos animales no humanos. Por ejemplo, en estudios donde un grupo de animales aprende una tarea determinada, después de alcanzar la asíntota de aprendizaje se sacrifica a los sujetos y se mide la activación neuronal en distintas áreas del cerebro. Los datos de este grupo de animales que aprendió la tarea se comparan luego con el nivel de activación neuronal en la misma región cerebral de otro grupo que, o bien no fue entrenado o recibió algún tipo de tratamiento control.

En peces existen antecedentes de estudios que utilizaron esta técnica histológica para observar la activación cerebral luego de una tarea espacial (e.g., Broglio, Rodríguez, Gómez, Arias \& Salas, 2010; Vargas, Rodríguez, López, Arias \& Salas, 2000). En estos casos, la tarea espacial consistió en colocar a los animales en un recipiente dentro del agua que tenía dos posibles salidas, pero una de ellas estaba bloqueada con un vidrio transparente. Los animales a lo largo de sucesivos ensayos, debían utilizar las claves espaciales disponibles para orientarse y aprender por cuál de las salidas era posible salir. Los sujetos del grupo control podían utilizar ambas salidas por lo que para ellos, no era requerida ninguna orientación espacial. Los resultados obtenidos por la técnica de AgNOR mostraron que en los sujetos experimentales una región lateral del telencéfalo, el pallium lateral (homóloga al hipocampo de los mamíferos; Northcutt, 1995, Northcutt \& Braford, 1980) presentaba un mayor tamaño relativo de los nucleolos con respecto a los sujetos controles, mientras que la región dorso medial no presentaba diferencias entre los grupos. Estos resultados coincidieron con estudios complementarios acerca de los efectos de lesiones practicadas en dicha área (el pallium lateral, región que presentaba mayor activación durante la tarea), ya que las mismas generaron un déficit en el desempeño de orientación en el espacio. De esta manera, la técnica de AgNOR aportó evidencias que señalaron que el pallium lateral de los peces, además de ser homólogo a la formación hipocampal de los mamíferos, presentaba cierto grado de equivalencia funcional.

En nuestro laboratorio recientemente hemos comenzado a utilizar esta técnica en el estudio de la actividad cerebral de los anfibios asociada a tareas espaciales y de aprendizaje aversivo. En este caso, es la región conocida como pallium medial del telencéfalo de los anfibios la que ha sido históricamente propuesta como homóloga del hipocampo de los mamíferos (Northcutt \& Ronan, 1992). A nivel funcional, hasta el momento se había observado que la lesión de esta área, del mismo modo que ocurre en mamíferos, aumentaba la resistencia a la extinción (Muzio, Segura \& Papini, 1993) y que también deterioraba la orientación en el espacio (Daneri, 2010; Daneri, Casanave \& Muzio, 2015). Actualmente aplicando la técnica de AgNOR, hemos verificado que el pallium medial también presenta un aumento de su activación durante una tarea espacial (Daneri, Puddington \& $\mathrm{Mu}$ zio, 2015). En cuanto al aprendizaje aversivo, como se mencionó anteriormente, es la amígdala una de las principales estructuras que participa del condicionamiento aversivo en mamíferos. En este caso, la utilización de la técnica de AgNOR en anfibios nos ha permitido observar que la región ventrolateral del telencéfalo, el striatum, que ha sido propuesta como homóloga de la amígdala de los mamíferos (Moreno \& González, 2007), incrementa su activación neuronal en una tarea de evitación pasiva, indicando también un alto grado 
de equivalencia funcional (Puddington, Daneri, Papini \& Muzio, 2015).

En mamíferos, la técnica de AgNOR ha sido aplicada en varias especies y con variados objetivos. Por ejemplo, García-Moreno, Cimadevilla, González-Pardo, Zahonero y Arias (1997) emplearon la técnica de AgNOR en ratas para un estudio del desarrollo del hipocampo. Observaron que en ratas jóvenes el volumen de los nucleolos tiende a incrementarse, mientras que en ratas adultas esta tendencia se revierte, sugiriendo que el envejecimiento está asociado a una menor actividad hipocampal. En un estudio posterior (García-Moreno et al., 2000) también se utilizó esta técnica en ratas para caracterizar la actividad basal de dos regiones del hipocampo, CA1 y CA3, ambas muy relevantes para el aprendizaje, observándose que eran las porciones dorsales de estas dos regiones las que presentaban una mayor actividad basal que las porciones ventrales. Así, estos resultados obtenidos con la técnica de AgNOR plantearon la necesidad de estudios futuros que puedan explicar más precisamente el significado comportamental y fisiológico de esta heterogeneidad funcional en la formación del hipocampo. Otro trabajo también utilizó esta técnica para caracterizar la actividad diferencial de distintas regiones del cerebro entre hembras y machos (González-González, Díaz, Vallejo \& Arias, 1996). Los resultados de este estudio indicaron que los cuerpos mamilares, una región basal del cerebro que también forma parte del sistema límbico y participa en los procesos de memoria, muestran una activación diferencial luego de una estimulación hormonal, indicando así la importancia de las hormonas sobre la actividad de síntesis de proteínas celulares. También hay dos investigaciones en ratas en las que se empleó la técnica de AgNOR para estudiar los cambios en la actividad cerebral luego de distintos tratamientos. En un caso, se observó un deterioro en la actividad del hipocampo y la corteza inferotemporal como consecuencia de una colestasis (i.e., la interrupción del flujo de la bilis del hígado al duodeno) producida mediante una intervención quirúrgica (García-Moreno et al., 2002). En el segundo caso se observó un deterioro de la actividad de las dos regiones hipocampales mencionadas anteriormente, CA1 y CA3, como consecuencia del consumo crónico de alcohol (García-Moreno et al., 2001).

Todos estos antecedentes muestran que la técnica de AgNOR puede ser empleada para estudiar distintas especies y para abordar problemas muy diversos en neurociencias.

\section{ANTECEDENTES DE ESTUdIOS EN HUMANOS UTILI- ZANDO LA TÉCNICA DE AGNOR}

Dado que para el estudio de la actividad neural todas las técnicas histológicas requieren la extracción de tejido cerebral para su análisis, ninguno de los casos enunciados en el apartado anterior podría ser llevado a cabo en humanos. Sin embargo, el estudio postmortem de la actividad neuronal del cerebro, puede ser revelador en la investigación sobre un fenómeno psicológico para el cual no se cuenta con modelos animales.

Por ejemplo, el estudio mediante la técnica de AgNOR de cerebros de pacientes que se habían suicidado, permitió determinar que el tamaño de los nucleolos en las neuronas del núcleo del rafe dorsal estaba aumentado con respecto al de sujetos controles sanos que murieron por otra causa. Llamativamente, también se pudo observar que los sujetos depresivos que murieron por una causa distinta al suicidio presentaban una menor actividad del núcleo del rafe dorsal con respecto a los suicidas, y aún menor que en los sujetos sanos (Bielau et al., 2005). Estos resultados coincidieron con los de un estudio posterior, que replicó la investigación en otros sujetos utilizando el mismo procedimiento (Gos et al., 2007a). También se analizó la actividad de las neuronas piramidales del cíngulo anterior de estos mismos sujetos. Se observó que los pacientes depresivos en general tenían una menor actividad de esta región en el hemisferio derecho. En cambio, en el hemisferio izquierdo sólo los pacientes suicidas presentaban un decremento en la activación (Gos et al., 2007b). Utilizando esta misma muestra se observaron también alteraciones de activi- 
dad en neuronas de la corteza orbitofrontal (Gos et al., 2009a) y del globo pálido (Gos et al., 2009b) entre los pacientes depresivos suicidas y no suicidas. Así, en todos estos estudios, el empleo de la técnica de AgNOR para medir la actividad celular de algunas regiones cerebrales en personas que presuntamente cometieron suicidio, es una herramienta potencialmente útil para el diagnóstico forense. Lamentablemente, los tamaños muestrales en esta serie de publicaciones son pequeños, por lo que se requerirán nuevos estudios para poder incorporar al AgNOR como técnica forense de rutina.

De todas formas, el conjunto de ejemplos presentados en esta revisión muestran el gran potencial que tiene esta técnica en el campo de la investigación en neurociencias, tanto en animales no humanos como en humanos.

\section{Conclusiones}

La técnica de AgNOR es una técnica histológica que permite cuantificar el nivel de síntesis de proteínas de una región cerebral. La información que proporciona esta técnica es por lo tanto útil para intentar responder distintos tipos de preguntas relevantes en neurociencias. Por ejemplo, ¿qué región cerebral presenta plasticidad neural luego de una tarea instrumental? También es posible indagar acerca de los cambios en el nivel de síntesis de proteínas que se dan en distintos momentos del desarrollo, o conocer qué regiones presentaban mayor activación en un paciente fallecido que padecía alguna enfermedad psicológica. Así, mediante la técnica de AgNOR es posible estudiar distintas partes del cerebro con funciones marcadamente diferentes y en variadas situaciones.

Existen también otras técnicas histológicas que permiten abordar preguntas de este estilo; principalmente, mediante técnicas inmunohistoquímicas (basadas en la identificación de genes de expresión temprana, que utilizan anticuerpos). Sin embargo, la técnica de AgNOR presenta algunas ventajas que vale la pena destacar. En primer lugar, los reactivos son notablemente más económicos en el caso de la tinción con nitrato de plata que en el caso de las técnicas que usan anticuerpos. Y en segundo lugar, la técnica de AgNOR es mucho más simple y por lo tanto, menos exigente con los procedimientos y cuidados que se deben tener con el tejido a estudiar. Pero sobre estas dos ventajas, es importante señalar que la técnica de AgNOR tiñe dos estructuras que están presentes en todas las células eucariotas, los núcleos y sus nucleolos. De esta forma, es potencialmente aplicable en todas las especies animales. De hecho, esta técnica ha mostrado ser útil en el estudio del cerebro de distintos grupos zoológicos: peces, anfibios y mamíferos.

El empleo de la técnica de AgNOR como complemento del estudio conductual no ha sido todavía valorado en su verdadera dimensión y se presenta como una herramienta útil para comprender los mecanismos que subyacen a los comportamientos. Su difusión dentro de los especialistas en ciencias de la conducta contribuirá a un mejor entendimiento de la relación entre la activación neuronal de distintas áreas cerebrales y las conductas asociadas. 
FIGURA 1

IMAGEN DE UN CORTE CORONAL DE LA CORTEZA ORBITOFRONTAL DE UNA RATA (IZQUIERDA) Y EL TELENCÉFALO MEDIO DE UN SAPO (DERECHA), CORRESPONDIENTE A LA TINCIÓN DE AGNOR (CON NITRATO DE PLATA)

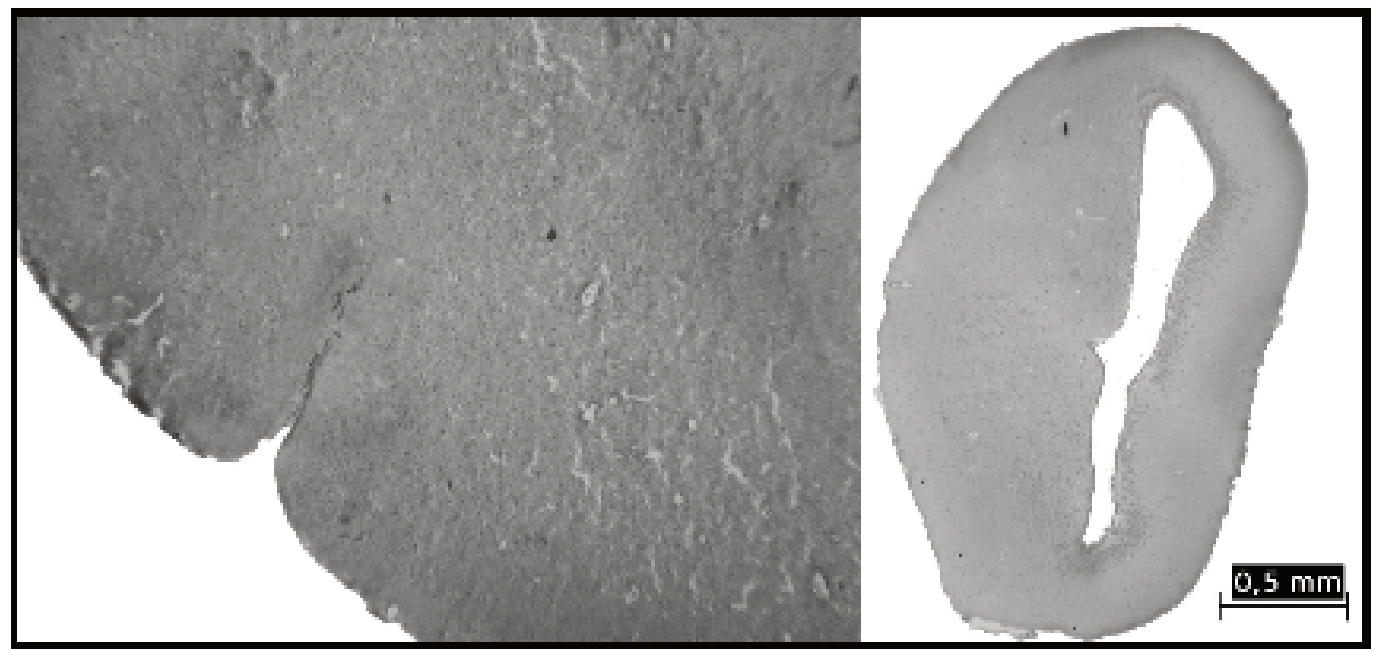

Nota:

Aumento $\times 40$

FIGURA 2

IMAGEN EN LA QUE SE OBSERVAN EJEMPLOS DE NÚCLEOS Y NUCLEOLOS DE NEURONAS TAL COMO PERMITE VISUALIZARLOS LA TINCIÓN DE AGNOR

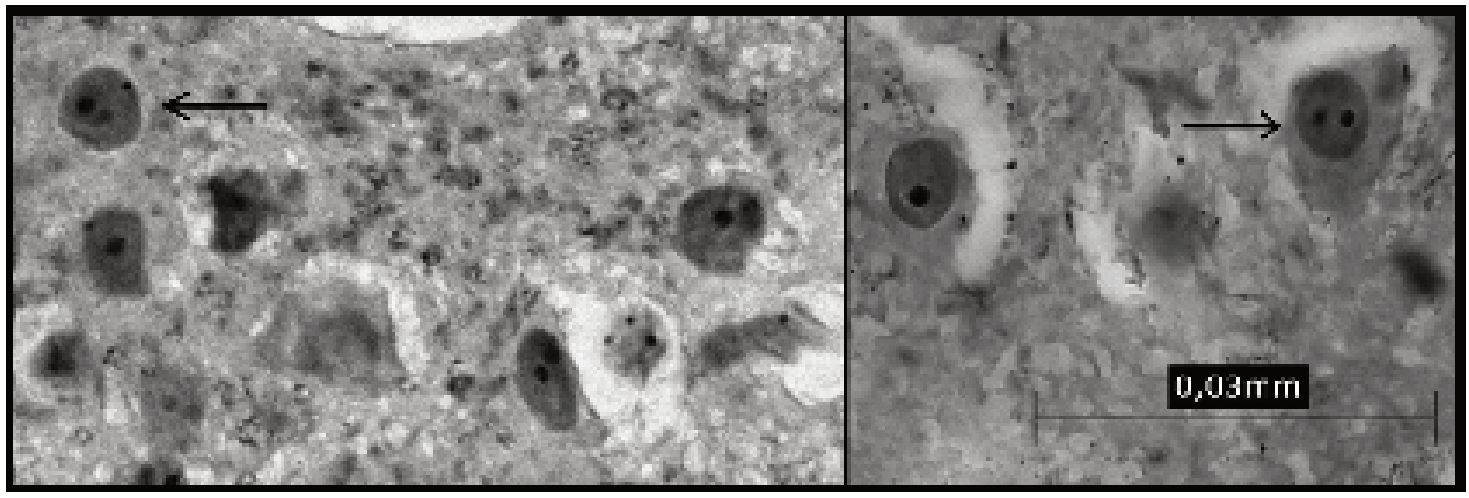

Nota:

La imagen de la izquierda corresponde a tejido de rata y la de la derecha, a tejido de sapo. En ambos casos la flecha marca un núcleo que presenta doble nucleolo.

Aumento x1000. 


\section{REFERENCIAS BIBLIOGRÁFICAS}

Alberts, B., Johnson, A., Lewis, J., Raff, M., Roberts, K. \& Walter, P. (2008). Molecular biology of the cell (5ta. ed.). New York: Garland Science.

Bandettini, P.A. (2009). What's new in neuroimaging methods? Annals of the New York Academy of Sciences, 1156, 260-293. http://dx. doi.org/10.1111/j.1749-6632.2009.04420.x

Bear, M.F., Connors, B.W. \& Paradiso, M.A. (2006). Neuroscience: Exploring the brain (2da. ed.). New York: Williams \& Wilkins.

Bielau, H., Mawrin, C., Krell, D., Agelink, M.W., Trübner, K., Davis, R., Gos, T., Bogerts, B., Bernstein, H.G. \& Baumann, B. (2005). Differences in activation of the dorsal raphe nucleus depending on performance of suicide. Brain Research, 1039(1), 43-52. http://dx.doi.org/1 0.1016/j.brainres.2005.01.055

Broglio, C., Rodríguez, F., Gómez, A., Arias, J.L. $\&$ Salas, C. (2010). Selective involvement of the goldfish lateral pallium in spatial memory. Behavioural Brain Research, 210(2), 191-201. http://dx.doi.org/10.1016/j.bbr.2010.02.031

Campese, V.D., Gonzaga, R., Moscarello, J.M. \& LeDoux, J.E. (2015). Modulation of instrumental responding by a conditioned threat stimulus requires lateral and central amygdala. Frontiers in Behavioral Neuroscience, 9, 293. http://dx. doi.org/10.3389/fnbeh.2015.00293

Campese, V.D., Kim, J., Lázaro-Muñoz, G., Pena, L., LeDoux, J.E. \& Cain, C.K. (2014). Lesions of lateral or central amygdala abolish aversive pavlovian-to-instrumental transfer in rats. Frontiers in Behavioral Neuroscience, 8,161 . http:// dx.doi.org/10.3389/fnbeh.2014.00161

Castellucci, V.F., Blumenfeld, H., Goelet, P. \& Kandel, E.R. (1989). Inhibitor of protein synthesis blocks longterm behavioral sensitization in the isolated gill withdrawal reflex of Aplysia. Journal of Neurology, 20(1), 1-9. http://dx. doi. org/10.1002/neu.480200102
Daneri, M.F. (2010). Aprendizaje espacial en el anfibio anuro Bufo arenarum. Estrategias, fenómenos y bases neurales [Spatial learning in the amphibian Bufo arenarum anuro. Strategies, phenomena and neural bases]. Tesis doctoral no publicada. Facultad de Ciencias Exactas y Naturales. Universidad de Buenos Aires.

Daneri, M.F., Casanave, E.B. \& Muzio, R.N. (2015). Medial Pallium lesion affects both turn and cue spatial learning in terrestrial toads (Rhinella arenarum). Manuscrito no publicado.

Daneri, M.F., Puddington, M.M. \& Muzio, R.N. (2015). Spatial learning induces increasing of argyrophilic nucleolar organizer region (AgNOR) of medial pallium neurons in toads (Rhinella arenarum). Manuscrito no publicado.

Edelman, G. M. \& Tononi, G. (2000). A universe of consciousness: How matter becomes imagination. New York: Basic Books.

Freeman, F., Rose, S.P.R. \& Sholey, A. (1995). Two time windows of anisomycin-induced amnesia for passive avoidance training in the day-old chick. Neurobiology of Learning and Memory, 63, 291-295. http://dx.doi.org/10.1006/nlme.19 95.1034

Frey, U., Krug, M., Reymann, K.G. \& Matthies, H. (1988). Anisomycin, an inhibitor of protein synthesis, blocks late phases of LTP phenomena in the hippocampal CA1 region in vitro. Brain Research, 452(1), 57-65. http://dx.doi.org/10. 1016/0006-8993(88)90008-X

García-Moreno, L.M., Aller, M.A., Conejo, N.M., Gómez, M.A., Martín, F.R., Arias, J. \& Arias, J.L. (2002). Brain AgNOR activity in cholestatic rats with hepatic encephalopathy. Hepatology Research, 24(3), 275-281. http://dx.doi. org/10.1016/S1386-6346(02)00132-8

García-Moreno, L.M., Cimadevilla, J.M., González-Pardo, H. \& Arias, J.L. (2000). Functional differences between dorsal and ventral hippocampus revealed with AgNOR staining. Psicothema, 12(2), 293-295. 
García-Moreno, L.M., Cimadevilla, J.M., González-Pardo, H., Zahonero, M.C. \& Arias, J.L. (1997). NOR activity in hippocampal areas during the postnatal development and ageing. Mechanisms of Ageing and Development, 97, 173-181. http://dx.doi.org/10.1016/S0047-637 4(97)00054-7

García-Moreno, L.M., Conejo, N.M., Pardo, H.G., Gómez, M., Martín, F.R., Alonso, M.J. \& Arias, J.L. (2001). Hippocampal AgNOR activity after chronic alcohol consumption and alcohol deprivation in rats. Physiology \& Behavior, 72 (1), 115-121. http://dx.doi.org/10.1016/S003 1- 9384(00)00408-X

González-González, S., Díaz, F., Vallejo, G. \& Arias, J.L. (1996). Functional sexual dimorphism of the nucleolar organizer regions in the tuberomamillary nucleus. Brain Research, 736(1), 1-6. http://dx.doi.org/10.1016/00068993(96)00602-6

Gore, F., Schwartz, E.C., Brangers, B.C., Aladi, S., Stujenske, J.M., Likhtik, E., Russo, M.J. Gordon, J.A., Salzman, C.D. \& Axel, R. (2015). Neural representations of unconditioned stimuli in basolateral amygdala mediate innate and learned responses. Cell, 162(1), 134-145. http:// dx.doi.org/10.1016/j.cell.2015.06.027

Gos, T., Krell, D., Bielau, H., Steiner, J., Mawrin, C., Trübner, K., Brisch, R., Bernstein, H.G., Jankowski, Z. \& Bogerts, B. (2009a). Demonstration of disturbed activity of orbitofrontal pyramidal neurons in depressed patients by the AgNOR staining method. Journal of Affective Disorders, 118(1), 131-138. http://dx.doi.org/ 10.1016/j.jad.2009.02.012

Gos, T., Krell, D., Bielau, H., Steiner, J., Trübner, K., Brisch, R., Bernstein, H.G., Jankowski, Z. \& Bogerts, B. (2009b). Demonstration of disturbed activity of external globus pallidus projecting neurons in depressed patients by the AgNOR staining method. Journal of Affective Disorders, 119(1), 149-155. http://dx.doi.org/ 10.1016/j.jad.2009.03.010
Gos, T., Krell, D., Brisch, R., Bielau, H., Trübner, K., Bernstein, H.G.\& Bogerts, B. (2007a). The changes in AgNOR parameters of dorsal raphe nucleus neurons are related to suicide. Legal Medicine, 9(5), 251-257. http://dx.doi.org/10. 1016/j.legalmed.2007.02.001

Gos, T., Krell, D., Brisch, R., Bielau, H., Trübner, K., Bernstein, H.G. \& Bogerts, B. (2007b). The changes of AgNOR parameters of anterior cingulate pyramidal neurons are region-specific in suicidal and non-suicidal depressive patients. The World Journal of Biological Psychiatry, 8(4), 245-255. http://dx.doi.org/10.10 80/15622970601169758

Harlow, J.M. (1868). Recovery from the passage of an iron bar through the head. Publications of the Massachusetts Medical Society, 2(3), 327-347. http://dx.doi.org/10.1177/ 0957154X 9300401407

Hernandez, P.J., Sadeghian, K. \& Kelley, A.E. (2002). Early consolidation of instrumental learning requires protein synthesis in the nucleus accumbens. Nature Neuroscience, 5(12), 1327-1331. http://dx.doi.org/10.1038/n n973

LeDoux, J.E. (2003). The emotional brain, fear, and the amygdala. Cellular and Molecular Neurobiology, 23(4-5), 727-738. http://dx.doi. org/10.1023/A:1025048802629

McWilliams, S.A. (2015). Psychology, history of psychology (Twentieth Century). En J.D. Wright (Ed.), International Encyclopedia of the Social \& Behavioral Sciences (2da. ed.), pp. 412-417. New York: Elsevier.

Moreno, N. \& González, A. (2007). Evolution of the amygdaloid complex in vertebrates, with special reference to the anamnio-amniotic transition. Journal of Anatomy, 211, 151-163. http://dx.doi.org/10.1111/j.1469-7580.2007.00 780.x

Muzio, R.N., Segura, E.T. \& Papini, M.R. (1993). Effects of lesions in the medial pallium on instrumental learning in the toad (Bufo arenarum). Physiology and Behavior, 54(1), 185- 
188. http://dx.doi.org/10.1016/0031-9384(93) 90 064-M

Nader, K., Schafe, G.E. \& LeDoux, J.E. (2000). Fear memories require protein synthesis in the amygdala for reconsolidation after retrieval. Nature, 406(6797), 722-726. http://dx.doi.org/ 10.1038/35021052

Naghdi, N., Majlessi, N. \& Bozorgmehr, T. (2003). The effects of anisomycin (a protein synthesis inhibitor) on spatial learning and memory in CA1 region of rats hippocampus. Behavioural Brain Research, 139(1), 69-73. http://dx.doi. org/10.1016/S0166-4328(02)00060-8

Northcutt, R.G. (1995). The forebrain of gnathostomes: In search of a morphotype. Brain, Behavior and Evolution, 46, 275-318. http://dx. doi.org/10.1159/000113280

Northcutt, R.G. \& Braford, M.R. (1980). New observations on the organization and evolution of the telencephalon of actinopterygian fishes. En S.O.E. Ebbesson (Ed.), Comparative neurology of the telencephalon (pp. 41-98). New York: Plenum. http://dx.doi.org/10.1007/9781-4613-2988-6 3

Northcutt, R.G. \& Ronan, M. (1992). Afferent and efferent connections of the bullfrog medial pallium. Brain, Behavior and Evolution, 40, 116. http://dx.doi.org/10.1159/000113898

Penfield, W. \& Rasmussen, T. (1950). Excision of cortical regions. Central sensorimotor cortex. En W. Penfield \& T. Rasmussen (Eds.), The cerebral cortex of man: A clinical study of localization of function (pp. 184-186). New York: Macmillan.

Ploton, D., Menager, M., Jeannesson, P., Himber, G., Pigeon, F. \& Adnet, J.J. (1986). Improvement in the staining and in the visualization of the argyrophilic proteins of the nucleolar organizer region at the optical level. Histochemical Journal, 18, 5-14. http://dx.doi.org/ 10.1007/BF01676192

Power, A.E., Berlau, D.J., McGaugh, J.L. \& Steward, O. (2006). Anisomycin infused into the hippocampus fails to block "reconsolidation" but impairs extinction: the role of reexposure duration. Learning and Memory, 13(1), 27-34. http://dx.doi.org/10.1101/lm.912 06.

Puddington, M.M., Daneri, M.F., Papini, M.R. \& Muzio, R.N. (2015). Telencephalic neural activation after passive avoidance learning in the terrestrial toad Rhinella arenarum. Manuscrito no publicado.

Purves, D., Augustine, G.J., Fitzpatrick, D., Hall, W.C., LaMantia, A.S., Mc Namara, J.O. \& Williams, S.M. (2004). The somatic sensory system. En D. Fitzpatrick (Ed.), Neuroscience. (Cap. 8, pp.189-208). Sunderland, MA: Sinauer Associates.

Quian Quiroga, R. \& Panzeri, S. (2009). Extracting information from neuronal populations: Information theory and decoding approaches. Nature Reviews Neuroscience, 10, 173-185. http://dx.doi.org/10.1038/nrn2578

Ramirez, F., Moscarello, J.M., LeDoux, J.E. \& Sears, R.M. (2015). Active avoidance requires a serial basal amygdala to nucleus accumbens shell circuit. The Journal of Neuroscience, 35 (8), 3470-3477. http://dx.doi.org/10.1523/JN EUROSCI.1331-14.2015

Raška, I., Koberna, K., Malínský, J., Fidlerová, H. \& Mašata, M. (2004). The nucleolus and transcription of ribosomal genes. Biology of the Cell, 96(8), 579-594. http://dx.doi.org/10101 6/j.biolcel.2004.04.015

Rodríguez, F., López, J.C., Vargas, J.P. \& Salas, C. (1998). Fundamentos de Psicobiología [Psychobiology foundations]. Sevilla: Kronos.

Schultz, W., Dayan, P. \& Montague, P.R. (1997). A neural substrate of prediction and reward. Science, 275(5306), 1593-1599. http://dx.doi. org/10.1126/science.275.5306.1593

Tilson, H.A. (2000). Neurotoxicology risk assessment guidelines: Developmental neurotoxicology. Neurotoxicology, 21, 189-194.

Titchener, E. (1909). Experimental psychology of the thought process. New York: Macmillan. 
Vargas, J.P., Rodríguez, F., López, J.C., Arias, J.L. \& Salas, C. (2000). Spatial learning-induced increase in the argyrophilic nucleolar organizer region of dorsolateral telencephalic neurons in goldfish. Brain Research, 865(1), 77-84. http:// dx.doi.org/10.1016/S0006-8993(00)02220-4

Watson, J.B. (1913). Psychology as the behaviorist views it. Psychological Review, 20(2), 158. http://dx.doi.org/10.1037/h0074428

Grupo de Aprendizaje y Cognición Comparada Laboratorio de Biología del Comportamiento Instituto de Biología y Medicina Experimental (IBYME) Consejo Nacional de Investigaciones Científicas y Técnicas (CONICET) Facultad de Psicología Universidad de Buenos Aires (UBA) Ciudad Autónoma de Buenos Aires - República Argentina

Fecha de recepción: 13 de agosto de 2015 Fecha de aceptación: 2 de marzo de 2016 